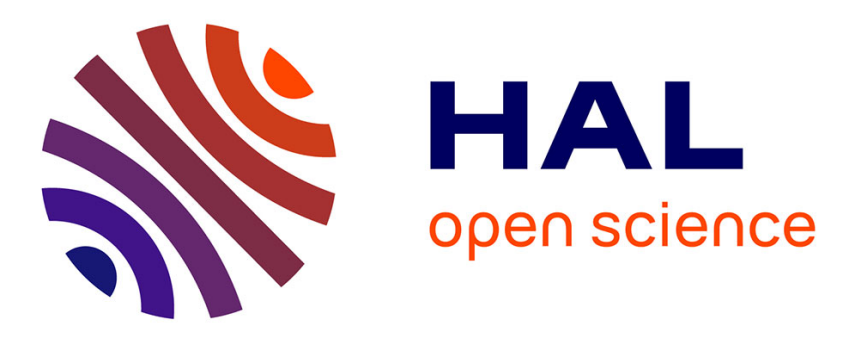

\title{
Quantitative cellular uptake of double fluorescent core-shelled model submicronic particles
}

Lara Leclerc, Delphine Boudard, Jérémie Pourchez, Valérie Forest, Laurence Marmuse, Cédric Louis, Valérie Bin, Sabine Palle, Philippe Grosseau, Didier Bernache-Assollant, et al.

\section{To cite this version:}

Lara Leclerc, Delphine Boudard, Jérémie Pourchez, Valérie Forest, Laurence Marmuse, et al.. Quantitative cellular uptake of double fluorescent core-shelled model submicronic particles. Journal of Nanoparticle Research, 2012, 14 (11), pp.14:1221. 10.1007/s11051-012-1221-x . hal-00757737

\section{HAL Id: hal-00757737 https://hal.science/hal-00757737}

Submitted on 28 Nov 2012

HAL is a multi-disciplinary open access archive for the deposit and dissemination of scientific research documents, whether they are published or not. The documents may come from teaching and research institutions in France or abroad, or from public or private research centers.
L'archive ouverte pluridisciplinaire HAL, est destinée au dépôt et à la diffusion de documents scientifiques de niveau recherche, publiés ou non, émanant des établissements d'enseignement et de recherche français ou étrangers, des laboratoires publics ou privés. 


\title{
Quantitative cellular uptake of double fluorescent core-shelled model submicronic particles
}

Lara Leclerc, Delphine Boudard, Jérémie Pourchez, Valérie Forest, Laurence Marmuse, Cédric Louis, Valérie Bin, Sabine Palle, Philippe Grosseau, Didier Bernache-Assollant, Michèle Cottier

\author{
L. Leclerc (Corresponding-Author), J. Pourchez, V. Forest, P. Grosseau, D \\ Bernache-Assollant \\ Ecole Nationale Supérieure des Mines, CIS-EMSE, LINA EA 4624, F-42023, \\ Saint-Etienne, France \\ e-mail: leclerc@emse.fr Phone: (+33) 477499700 Fax: (+33) 477499694
}

D. Boudard, V. Bin, M. Cottier

LINA EA-4624, F-42023, Saint-Etienne, France

L. Marmuse, C. Louis

NANO-H S.A.S., 2 place de l'Europe, Bâtiment A, Parc d'activité GVIO, F-38070, Saint Quentin Fallavier, France

\section{S. Palle}

Université Jean Monnet, Centre de Microscopie Confocale Multiphotonique, Pôle Optique et Vision, F-42023, Saint-Etienne, France

\footnotetext{
Abstract Introduction: The relationship between particles' physicochemical parameters, their uptake by cells and their degree of biological toxicity represent a crucial issue, especially for the development of new technologies such as fabrication of micro- and nanoparticles in the promising field of drug delivery systems. This work was aimed at developing a proof-of-concept for a novel model of double fluorescence submicronic particles that could be spotted inside phagolysosomes.

Materials and methods: Fluorescein isothiocyanate (FITC) particles were synthesized and then conjugated with a fluorescent pHrodo $^{\mathrm{TM}}$ probe whose red fluorescence increases in acidic conditions such as within lysosomes. After validation in acellular conditions by spectral analysis with confocal microscopy and dynamic light scattering, quantification of phagocytosis was
} 
conducted on a macrophage cell line in vitro. The biological impact of pHrodo functionalization (cytotoxicity, inflammatory response and oxidative stress) was also investigated.

Results/Conclusion: Results validate the proof-of-concept of double fluorescent particles (FITC + pHrodo), allowing detection of entirely engulfed pHrodo particles (green and red labeling). Moreover incorporation of pHrodo had no major effects on cytotoxicity compared to particles without pHrodo, making them a powerful tool for micro- and nanotechnologies.

Keywords Fluorescent submicronic particles, cellular uptake, pHrodo, toxicity.

\section{Introduction}

Nanotechnology is an emerging multidisciplinary field that involves the synthesis of nanoscale materials (i.e., $10^{-9} \mathrm{~m}$ size range). The development of nanoparticles currently represents a significant issue for environmental sciences, biosciences and nanomedicine (Oberdorster et al. 2005). The specific properties of nanoparticles based on their physiochemical features offer potential application domains (Broz et al. 2006; Nan et al. 2008). So it is now generally recognized that ongoing assessments of the human health and ecological implications of exposure to nanoscale materials are necessary prerequisites before the commercial benefits of this technology can be fully realized (Warheit 2010).

Evaluating the potential hazards related to nanomaterials has become an emerging area in toxicology and health risk assessment (Clift et al. 2008; Fubini 1998; Fubini et al. 2004; Sayes et al. 2007; Warheit et al. 2004). Particle surfaces and interfaces are important components of nanoscale materials (Warheit 2008). It has been demonstrated in the literature that nanoparticles' toxicological effects are complex and involve a variety of factors, with physicochemical characteristics being chief among them (Warheit 2010; Warheit et al. 2007). Consequently, the 
physicochemical characteristics of the fluorescent particles developed in this study (i.e., size and chemical coating) were well-controlled; in particular all steps of particle synthesis were described and surface functionalization was homogeneous, preventing agglomeration phenomena. Controlling the physicochemical parameters was one of the main goals in particle specifications because it offers the possibility to functionalize them with other surface groups and different surface charges.

Regardless of the route of exposure (skin, digestive tract, respiratory tract or by injection), phagocytic cells such as macrophages are one of the main cells to respond. Indeed they are sentinel cells of the immune system and their main role consists of the internalization and destruction of foreign bodies by phagocytosis. Macrophages are model cells widely used in toxicological evaluations (Bruch et al. 2004; Clift et al. 2008; Fubini et al. 2004; Hamilton et al. 2008; Sayes et al. 2007).

The level of biological effects may be influenced by the amount of internalized particles in macrophages (Cho et al. 2009; Nan et al. 2008). Different metrical systems could be used to predict cellular response, such as surface, volume or number of particles. The mass of the material is the least appropriate metric, yet it is the easiest to measure (Fubini et al. 2010). The number of particles might be a more appropriate metric for uptake evaluations; however, it remains difficult to precisely quantify this amount within intracellular compartments (Bos and de Souza 2000; Leclerc et al. 2010).

During phagocytosis, foreign particles are internalized by vesicles called phagosomes, which fuse with lysosomes, another type of intracellular acidic vesicle, to form phagolysosomes. The $\mathrm{pH}$ is around 6 in a phagosome and decreases to 4.5 in a fully matured phagolysosome, which is a hydrolase-rich 
killing device (Griffiths 2004; Vieira et al. 2003). We took advantage of this property of acidification and developed double fluorescent particles sensitive to environmental $\mathrm{pH}$ changes that can be used as a tool for phagocytosis quantification. For this purpose and, to our knowledge, for the first time in the literature, we coupled FITC fluorescent particles with the $\mathrm{pHrodo}^{\mathrm{TM}}$ Succinimidyl ester probe (Invitrogen) (Harvey et al. 2008; Kobayashi et al. 2010; Miksa et al. 2009; Moore et al. 2008; Strunnikova et al. 2009). The red fluorescence of this probe becomes more intense with acidification and accurately indicates phagolysosomal localization.

Thus, our system directly detects particles engulfed in phagolysosomes, unlike existing systems described in the literature such as PEBBLE (Buck et al. 2004; Clark et al. 1999; Lee et al. 2009) and capsules (fluorescent microcapsules) (Reibetanz et al. 2010; Reibetanz et al. 2007; Romero et al. 2010). These microor nanostructures coupled with molecules such as SNARF dye (Munoz Javier et al. 2008; Semmling et al. 2008) function as $\mathrm{pH}$ sensors. Indeed, intracellular $\mathrm{pH}$ plays a key role in the regulation of different cellular processes (e.g. apoptosis, phagocytosis, cell growth, metabolism). It must be maintained at an optimal value, and knowledge of its alteration in complex biological samples is crucial. Moreover, there is a clear need for $\mathrm{pH}$-monitoring strategies that are noninvasive, sensitive, quantitative, stable and reproducible and that could be applied in realtime conditions (Peng et al. 2007). Systems such as PEBBLE and capsules were developed for this purpose, but they were not suitable for our purpose. The major problem with the capsules is their micrometric size (Sjöback et al. 1995); these systems were mainly developed for drug delivery system applications. PEBBLE systems were also not appropriate for our study because we did not want to monitor precise $\mathrm{pH}$ changes during the phagocytic process, but rather localize 
particles in any type of acidic compartment. Moreover, particles' sizes and surface charges need to be variable, but it is difficult to control these factors for PEBBLEs during the synthesis process, which is a crucial step for specific study of cellular uptake.

Finally, we investigated the innocuousness of the particles' functionalization with the pHrodo probe and made comparisons between particles of equivalent sizes with and without pHrodo. The biological effects were evaluated based on inflammatory response assessed by tumor necrosis factor (TNF)- $\alpha$ production, integrity of the cell membrane measured by lactate dehydrogenase (LDH) release and oxidative stress following production of reactive oxygen species (ROS) (Bruch et al. 2004; Catelas et al. 1999; Donaldson et al. 2002; Sayes et al. 2007).

This study was conducted in two main parts. First, 250 and $500 \mathrm{~nm}$ fluorescent particles were synthesized, with or without incorporation of the $\mathrm{pH}$ sensitive probe pHrodo. These particles, which exhibited specific carboxylate functionalization, were characterized, and their fluorescence emission spectra were validated in acellular conditions. The second part of the study was a proofof-concept for these double fluorescent particles being able to detect particle phagocytosis after contact with macrophages.

\section{Experimental}

\section{Fluorescent particle development}

Tetraethyl orthosilicate $\left(\mathrm{Si}\left(\mathrm{OC}_{2} \mathrm{H}_{5}\right)_{4}, \quad\right.$ TEOS, $\left.\geq \quad 99 \%\right)$, (3aminopropyl)triethoxysilane $\left(\mathrm{H}_{2} \mathrm{~N}\left(\mathrm{CH}_{2}\right)_{3}-\mathrm{Si}\left(\mathrm{OC}_{2} \mathrm{H}_{5}\right)_{3}\right.$, APTES, 99\%), isopropanol $\left(\left(\mathrm{CH}_{3}\right)_{2} \mathrm{CHOH}, \geq 99.5 \%\right)$, triethylamine $\left(\left(\mathrm{C}_{2} \mathrm{H}_{5}\right)_{3} \mathrm{~N}, \geq 99.5 \%\right.$, dimethyl sulfoxide anhydrous $\left(\left(\mathrm{CH}_{3}\right)_{2} \mathrm{SO}, \mathrm{DMSO}, \geq 99.9 \%\right)$ fluorescein isothiocyanate isomere I 
$\left(\mathrm{C}_{21} \mathrm{H}_{11} \mathrm{NO}_{5} \mathrm{~S}\right.$, FITC, $\left.\geq 90 \%\right)$ and sodium hydroxide $(\mathrm{NaOH})$ were purchased from Aldrich Chemical (Sigma-Aldrich Chimie, Lyon, France). Diethylenetriamine pentaacetic dianhydride $\left(\mathrm{C}_{14} \mathrm{H}_{19} \mathrm{~N}_{3} \mathrm{O}_{8}\right.$, DTPA-ba, > 98.0\%) was purchased from TCI Europe (Zwijndrecht, Belgium). pHrodo succinimidyl ester (pHrodo, SE) was purchased from Invitrogen (Cergy Pontoise, France). Ethanol, diethylene glycol (DEG, 99\%) and other organic solvents (reagent grade) were purchased from SDS (France) and used as received.

Two types of fluorescent particles with variable and well-defined sizes (250 and $500 \mathrm{~nm}$ ) were developed and produced (Nano-H, France). The particles were composed of pure silica core doped with FITC (green fluorescence) grafted by covalent bonds, as described by Van Blaaderen and Vrij (1992) and Wang et al. (2003). A controlled polysiloxane shell ( $\mathrm{SiOx}$ ) was added by hydrolysis condensation of APTES and TEOS, using isopropanol as a solvent in the presence of a catalytic amount of triethylamine (Fizet et al. 2009). During this step, functionalization using the pHrodo probe was performed by adding in desired percentages of the reaction product for the coupling between the dye using APTES in DMSO. The amorphous and porous properties of the polysiloxane shell allowed the probe to have contact with the $\mathrm{pH}$ variations. Finally the particles were stabilized by introducing carboxylic surface groups $(\mathrm{COOH})$ onto their surface using diethylenetriamie pentaacetic dianhydride DTPA-ba as the reagent. The synthesis was done as follows. DTPA-ba (1 equivalent/APTES) was stirred in DMSO $(15 \mathrm{mg} / \mathrm{mL})$ at room temperature for 15 minutes. A colloidal solution of particles in DMSO $(25 \mathrm{mg} / \mathrm{mL})$ was then added to the reaction and stirred for 18 hours at room temperature. A purification step was done by an initial centrifugation at $7000 \mathrm{rpm}$ for 10 minutes followed by resuspension of the precipitate in ethanol and a subsequent centrifugation (3 times, $7000 \mathrm{rpm}$ for 10 
minutes). The particles were then resuspended in Milli-Q water at the desired concentration (particle number/mL) at $\mathrm{pH} 9$.

Thus, two types of particles were synthesized:

- FITC particles exhibiting only a green fluorescence,

- FITC/pHrodo particles exhibiting double fluorescence (green/red), including red fluorescence of pHrodo probe, which increases as $\mathrm{pH}$ decreases, allowing the detection of engulfed intra-lysosomal particles.

The fluorescence of the particles was optimized by testing different percentages of dyes (Supplementary Table), ranging from $1.23 \%$ to 5\% (\% $\mathrm{m} / \mathrm{mSiO}_{2}$ ) for FITC grafting into the silica core and $0.1 \%$ to $2 \%$ for pHrodo grafting into $\mathrm{SiOx}(\% \mathrm{n} / \mathrm{nSiOx})$. Excitation and emission data of the dyes were $488 \mathrm{~nm}$ and $495-550 \mathrm{~nm}$ for FITC and $532 \mathrm{~nm}$ and $560-650 \mathrm{~nm}$ for pHrodo, respectively.

\section{Particle characterization}

Size distribution and zeta potential were measured using dynamic light scattering (DLS) with a Zetasizer Nano S from Malvern instrument (Orsay, France). Three measurements of the colloidal solution were taken directly after surface modification in water. Analyses were also carried out in complete culture media.

Transmission electron microscopy (TEM) observations were performed. Samples were prepared by diluting a solution of particles and depositing a drop of the solution on a copper grid coated with a thin film of amorphous carbon and allowing the liquid to air dry at room temperature. Images were acquired with a transmission electron microscope (Jeol 2010, Tokyo, Japan) operating at $200 \mathrm{kV}$. 
The fluorescence specificity of the particles was determined by confocal spectral analysis in acellular conditions. Analyses were performed using a confocal microscope TCS-SP2 from Leica Microsystems (Leica®, Heidelberg, Germany) coupled to an inverted microscope with oil immersion objective (HCX APO L U-V-I 63x1.2NA), and fitted with Acousto-Optical Beam Splitter (AOBS) covering a 350-850 $\mathrm{nm}$ wavelength range. The system was equipped with a set of argon $(458,476,488$ and $514 \mathrm{~nm})$, helium-neon $(543 \mathrm{~nm})$, helium-neon $(633 \mathrm{~nm})$

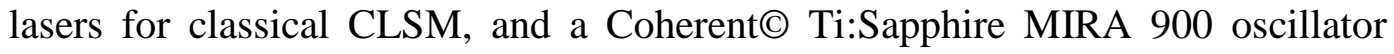
pumped with a $5 \mathrm{~W}$ Verdi Nd:YAG (532 nm) laser. The femtolaser system typically delivered $800 \mathrm{~mW}, 200$ fs pulses with a $76 \mathrm{MHz}$ repetition rate. Suspensions of particles were placed in appropriate cup-shaped (four wells LabTek $^{\mathrm{TM}}$ Chamber Slides, Nunc $\left.{ }^{\circledR}\right)$ and emission fluorescence spectral curves were collected with $10 \mathrm{~nm}$ resolution over the wavelength range of 400 to $800 \mathrm{~nm}$ using two-photon excitation and AOBS. For FITC/pHrodo particles, acquisitions were done in buffer solutions of different $\mathrm{pH}$ values ( $\mathrm{pH} 4$ and $\mathrm{pH} 10)$.

\section{Macrophage cell culture}

The RAW 264.7 cell line was provided by ATCC Cell Biology Collection (Promochem LGC, Molsheim, France) and was derived from murine peritoneal macrophages transformed by the Abelson murine leukemia virus. Cells were cultured in Dulbecco's modified Eagle's medium (DMEM, Invitrogen), complemented with $10 \%$ of fetal calf serum (FCS, Invitrogen), $1 \%$ penicillinstreptomycin (penicillin 10,000 U/mL, streptomycin $10 \mathrm{mg} / \mathrm{mL}$; Sigma-Aldrich, Saint-Quentin Fallavier, France) and incubated at $37^{\circ} \mathrm{C}$ under a $5 \% \quad \mathrm{CO}_{2}$ humidified atmosphere. 
The particles' fluorescence was assessed after a 24 hour incubation with macrophages using an arbitrary ratio of 1000 particles for one cell (1000/cell) based on the macrophage size compared to the particle sizes. Particles were strongly vortexed for 30 seconds before use.

\section{Treated cells electron microscopy}

Cells were grown in six-well plates (1 million cells in $4 \mathrm{~mL}$ of complete culture medium) containing coverslips. Particles were incubated for 24 hours.

For scanning electron microscopy (SEM) observations, samples were fixed for 15 minutes with $2.5 \%$ glutaraldehyde (Sigma) and dehydrated in acetone baths of increasing concentrations $(30 \%, 50 \%, 70 \%, 80 \%, 90 \%, 95 \%$ and 99\%, 10 min/bath) (Di Palma et al., 2004). Coverslips were sputtered coated with gold and examined with a FEG-SEM (Field Emission Gun, 6500F Jeol, Japan).

For TEM observations, samples were fixed in $1 \%$ glutaraldehyde/ $0.5 \%$ paraformaldehyde in $0.1 \mathrm{M}$ mono $\mathrm{Na} / \mathrm{diK}$ buffer ( $\mathrm{pH} 7.4$ ), postfixed in $1 \%$ osmium tetroxide in $0.1 \mathrm{M}$ cacodylate buffer for 1 hour, dehydrated in a graded series of ethanol and embedded in Epon resin. Ultrathin $(70 \mathrm{~nm})$ sections were cut and stained with uranyl acetate and lead citrate. Images were acquired with a transmission electron microscope (MET Hitachi H800-3, Tokyo, Japan) equipped with a CCD camera (XR40; AMT).

\section{Confocal imaging and phagocytosis semi-quantification}

Cells were grown in six-well plates (1 million cells in $4 \mathrm{~mL})$ containing coverslips. Particles were incubated for 24 hours. Wells were fixed with $4 \%$ paraformaldehyde for 10 minutes and washed with phosphate-buffered saline 
(PBS, VWR, Fontenay sous bois, France) in order to remove nonadherent or noninternalized free particles that remained in the supernatant. However, this wash did not remove particles adhered to the cell membrane. Finally cell nuclei were stained with $10 \mu \mathrm{M}$ of Hoechst 33342 solution (Sigma) for 15 minutes and rinsed with PBS. Images were obtained from the fluorescence (excitation, emission) emitted by FITC (488 nm, 495-550 nm), pHrodo (532 nm, 560-650 $\mathrm{nm})$ and Hoechst $(350 \mathrm{~nm}, 408-443 \mathrm{~nm})$. Results are expressed as means of the fluorescence of acquired image stacks. Three independent analyses were performed in duplicate.

Stacks obtained by confocal imaging (green and red fluorescence) were treated separately with a computer interface $($ Matlab®). Stacks were transposed to grey levels followed by an automatic thresholding allowing obtention of arbitrary values for the number of green and red pixels. A ratio was then established between red and green pixels, leading to a percentage of internalized particles.

\section{Toxicity assessment}

For each experiment, cells were prepared in $96-$ well plates $(100,000$ cells/well for TNF- $\alpha$ and LDH assays, and 300,000 cells/well for ROS and $\mathrm{H}_{2} \mathrm{O}_{2}$ measurements) in $25 \mu \mathrm{L}$ of complete DMEM as previously described (Leclerc et al. 2010). Particles (1000/cell in a volume of $75 \mu \mathrm{L}$ of DMEM) were then added to the culture and incubated for 24 hours at $37^{\circ} \mathrm{C}$ in a $5 \% \mathrm{CO}_{2}$ atmosphere. In each case, control cells were incubated in the same conditions but without particles.

TNF- $\alpha$ production was assessed by a commercial ELISA Kit (Quantikine ${ }^{\circledR}$ Mouse TNF- $\alpha$ Immunoassay, R\&D Systems, Lille, France) according to the manufacturer's instructions. The optical density of each well was determined using a microplate reader (Multiskan RC, Thermolabsystems, Helsinki, Finland) 
set to $450 \mathrm{~nm}$. A standard curve was established and results were expressed in picograms of TNF- $\alpha$ per milliliter of supernatant.

The activity of the LDH released from cells with damaged membranes was assessed using the CytoTox-ONE ${ }^{\mathrm{TM}}$ Homogeneous Membrane Integrity Assay (Promega, Charbonnières les bains, France) according to the manufacturer's instructions. Detection was performed with a fluorometer (Fluoroskan Ascent, Thermolabsystems), using excitation/emission wavelengths at 530/590 nm. The activity of the released LDH was reported in comparison to that of total cellular LDH (measured after the lysis of control cells) and was expressed as a percentage of the control.

Concerning oxidative stress, ROS activity was assessed with the OxiSelect ${ }^{\mathrm{TM}}$ ROS Assay Kit (Euromedex, Mundolsheim, France). The assay is based on the conversion of a nonfluorescent substrate, 2',7'-dichlorodihydrofluorescin diacetate (DCFH-DA), which can easily diffuse through cell membranes, into a fluorogenic molecule, 2',7'-dichlorodihydrofluorescein (DCF), which is highly fluorescent and whose amount is proportional to total ROS. DCF production was detected with a Fluoroskan Ascent fluorometer (Thermolabsystems) using excitation and emission wavelengths of $480 \mathrm{~nm}$ and $530 \mathrm{~nm}$, respectively, and expressed as nanomoles per hour.

Finally, the release of hydrogen peroxide $\left(\mathrm{H}_{2} \mathrm{O}_{2}\right)$ was measured as described by De la Harpe and Nathan (1985). After incubation, cells were stimulated to release $\mathrm{H}_{2} \mathrm{O}_{2}$ produced by addition of $100 \mathrm{ng} / \mathrm{mL}$ phorbol-12-myristate-13-acetate (Sigma). Briefly, Krebs-Ringer phosphate glucose buffer containing a mixture of scopoletin $(30 \mu \mathrm{M}), \mathrm{NaN}_{3}(1 \mathrm{mM})$ and horseradish peroxidase $(1 \mathrm{U}$ pupurogallin/mL HPO) was added to the cells. The horseradish peroxidase catalyzed the oxidation of the fluorescent scopoletin, which was measured over a 
period of 90 minutes (Fluoroskan Ascent, Thermolabsystems) by using excitation/emission wavelengths at 355/460 nm. Results are expressed as means of arbitrary units of fluorescence.

\section{Statistical analysis}

Analysis and graphics were performed using Prism 5.0 software (GraphPad®, San Diego, CA, USA). Significance was established with ANOVA test $(p<0.05)$.

\section{Results and discussion}

\section{Particle development and characterization}

This study was aimed at developing a new concept of double fluorescent particles for biological investigations that would be particularly useful for the study of phagocytosis. pHrodo probes, whose fluorescence intensity increases as $\mathrm{pH}$ decreases, were functionalized in particle porous shells. A schematic representation of the two types of fluorescent particles developed is presented in Fig. 1. FITC particles exhibited green fluorescence only, while FITC/pHrodo particles emitted either a green or a yellow fluorescence. The latter color was observed in acidic conditions and indicated the colocalization of both green and red fluorescences (the red fluorescence of pHrodo appearing as $\mathrm{pH}$ declines).

DLS and TEM characterization were then performed in order to validate the synthesis of standardized particles, and to control size, shape and agglomeration in the tested media. Results are reported in Table 1, and an example of TEM acquisition is given in Fig. 2a. Globally, the particle sizes measured by DLS and TEM were in good agreement with the expected sizes (250 and $500 \mathrm{~nm}$, respectively). Size distributions in deionized water and in culture media were 
quite similar, with a slight increase in culture media possibly due to adsorption of proteins or other biomolecules at the surface of the particles. Moreover these particles appeared to be discrete on images. There was no evidence of agglomeration, even in presence of FCS, possibly because particles were produced directly in suspension, thus avoiding problems due to the dry-wet transition of particle powders, and because of the high stability of the particles allowed by the homogeneity of the $\mathrm{COOH}$ groups at their surface. These data were strongly supported by the zeta potential values, which were always negative despite significantly increasing in complete culture media. However, for the various types of particles, zeta potential values were quite similar in water and in complete culture media, suggesting that surface chemistry of the particles was well-controlled.

Concerning the optimization of dye amount, fluorescence intensity and dye stability (photobleaching) were checked under the confocal beam. For FITC particles, due to the fluorescence extinction, the best yield was obtained for $2 \%$ FITC. For the FITC/pHrodo particles, the best ratio was $1.23 \%$ FITC to $2 \%$ pHrodo. The adjustment of the percentages of dyes was an essential step in finding the optimal ratios, which were then used for the whole study (Supplementary Table).

The fluorescence emission spectra were then analyzed in acellular conditions in order to validate this innovative concept of double fluorescent particles. Fluorescence emission spectra ranging from 400 to $800 \mathrm{~nm}$ were measured by confocal microscopy. We confirmed that emission spectra fit adequately with the dyes used in the two types of particles. Fig. 3a shows the spectrum for $500 \mathrm{~nm}$ particles with 2\% FITC. Since FITC was deeply anchored, the fluorophore was not subjected to $\mathrm{pH}$ variations. The graphic presents a well-defined peak 
characteristic of the FITC emission. To verify the pHrodo probe's functioning, the emission spectra of FITC/pHrodo particles were determined either in a basic solution $(\mathrm{pH} \mathrm{10)}$ or in an acid solution $(\mathrm{pH} 4)$ and are reported in Fig. $3 \mathrm{~b}$ and 3c. At pH 10 (Fig. 3b), only one peak was observed, confirming the specificity of FITC fluorescence. Moreover, the shape of this spectrum was similar to that of simple FITC particles (Fig. 3a). However, a significant difference in FITC fluorescence intensity was observed due to the difference in FITC percentage (2\% vs. 1.23\%) between FITC particles and FITC/pHrodo particles. At pH 4 (Fig. 3c), two peaks specific to FITC and pHrodo probe, respectively, could be clearly distinguished, confirming the concept of the double fluorescence of these particles in acidic conditions.

As expected, the spectrum of FITC particles was specific to FITC fluorescence, while the spectrum of FITC/pHrodo particles was specific to FITC with the pHrodo probe-specific fluorescence becoming apparent in acidic conditions. It has already been described in the literature, especially by Peng et al. (2007), that FITC could be $\mathrm{pH}$ sensitive and therefore, FITC is sometimes directly used to monitor pH changes (Reibetanz et al. 2007; Skirtach et al. 2006). It is known that weak pH values $(<4)$ lead to a FITC fluorescence decrease. In this study, as previously mentioned, FITC was deeply anchored in the core-shell of the particles with no access to the external environment. The chemical manufacturing of the particles was successfully adapted to biological applications, especially to confocal microscopy. Indeed, we did not want to precisely monitor $\mathrm{pH}$ modifications, rather our aim was to track particles engulfed in acidic compartments. In this context, the appearance of the pHrodo red fluorescence was the determining parameter. 


\section{Cellular assays}

Once the particles' features were characterized, cellular assays were performed. The RAW 264.7 macrophage cell line was used as a representative model of the pulmonary cellular environment that particles are likely to interact with in vivo.

Interactions between macrophages and particles were observed using SEM. As illustrated in Fig. 2b, this technique allowed us to highlight the behavior of macrophages in contact with particles and to confirm their interactions, particularly at the level of the macrophage podosomes (white arrows).

After 24 hour contact with macrophages, $500 \mathrm{~nm}$ FITC particles were clearly visible on TEM images (Fig. 2c and 2d), which confirmed the uptake of particles by a phagocytic process. Once captured by macrophage podosomes, particles were surrounded by internalization vesicle membranes. Particles showed a cytoplasmic localization in vesicles with a double membrane in agreement with phagolysosome morphology. Similar results were found for the $250 \mathrm{~nm}$ FITC particles (Supplementary Fig. 1).

\section{Confocal imaging and phagocytosis semi-quantification by image analysis}

The particles' fluorescence was assessed after 24 hour incubation with macrophages using an arbitrary ratio of 1000 particles per one cell based on the macrophage size compared to the particles' size, in agreement with a previous study (Leclerc et al. 2010). Confocal microscopy was used to distinguish and quantify fluorescent particles that were entirely engulfed in acidic vesicles (yellow fluorescence) as opposed to those not internalized (green fluorescence). The double fluorescent $500 \mathrm{~nm}$ FITC/pHrodo particles were detectable by the red and green fluorescence spectra, as demonstrated by Fig. 3b and 3c. The merge shown 
in Fig. 4 allowed us to discriminate external particles (FITC green fluorescence) from particles internalized in acidic vesicles (yellow due to the green FITC and red pHrodo probe fluorescence colocalization). Therefore, these $\mathrm{pH}$-sensitive particles in confocal microscopy allowed discrimination of the particles' compartmentalization (inside/outside), and they represent a powerful tool to assess the phagocytic process of particles in macrophages. All the appropriate measures were taken to avoid cross-talk between the channels. Similar results were observed for $250 \mathrm{~nm}$ particles with and without pHrodo (Supplementary Fig. 2).

For phagocytosis quantification, stacks of images obtained by confocal microscopy (green and red fluorescences of the particles) were treated separately with a computer interface and were transposed to grey levels followed by an automatic thresholding algorithm allowing expression of the number of green and red pixels in arbitrary units. A ratio was then established between red and green pixels leading to a percentage of internalized particles. We found that after 24 hour incubation, $89 \pm 2 \%$ of the $250 \mathrm{~nm}$ particles and $86 \pm 5 \%$ of the $500 \mathrm{~nm}$ particles were internalized. This suggests that approximately $10 \%$ to $15 \%$ of the particles remained attached to cell membranes. Image analysis allowed us to establish a proportion of internalized particles. These data are semi-quantitative, but in good agreement with image observations and proved useful for determining the ratio between internalized and external particles.

We found no significant differences of cellular uptake between the two particle sizes investigated (250 and $500 \mathrm{~nm}$ ). This lack of difference could be due to the 24 hour time saturation of internalization. To that purpose, different exposure durations such as 6 hours and 12 hours should be tested to investigate kinetics of internalization. The same amount of internalization seems to occur independently 
for the two studied sizes. A larger range of particle sizes should be evaluated to confirm these observations and more particularly use particles $<100 \mathrm{~nm}$ to compare submicronic particle to nanoparticle uptake. Moreover, various parameters can be modified, e.g., particles/cell ratios or surface group functionalizations. This innovative approach will be of great use for the further investigation of particle cellular uptake quantification.

Recent studies have identified that the size, shape and surface properties are important factors in cellular uptake of nanoparticles (Chithrani, 2011). The surface ligand and charge of particles play an important role in their uptake process as well. The surface of the particles can be coated with different proteins for effective targeting to specific organelles. These data are very important for cancer cell targeting (Subramani et al. 2009, Abedini et al. 2011) or in the gene delivery field. For example, studies report the generation of "DNA nanoparticles" using polycations, which enhance the transfection efficiency (Hosseinkhani et al. 2006, 2008). Indeed, the uptake efficiency seems significantly affected by the surface charge of the particles: positively charged particles are more likely to be internalized than neutral or negatively charged particles (Cho et al. 2009), probably due to the cell membranes themselves being negatively charged. However, interactions with some cell surface molecules may be responsible for the facilitated uptake of negatively charged particles. It is also important to mention that surface charge and size of particles can play a major role in protein conjugation onto particle surfaces and this in turn influences the way the particles interact with cells and tissues.

These findings provide useful information to tailor nanoscale devices at the single-cell level for effective applications in diagnostics, therapeutics and imaging. It is very important to consider size, shape and surface properties when 
designing vectors for their applications in biomedical fields. These observations will be taken into account for the development of the FITC-pHrodo particles of different sizes and functionalized with different surface charges.

\section{Toxicity assessment}

Finally, a toxicological evaluation was performed on macrophages after 24 hour contact with particles to assess the use of these particles in biological conditions, and more particularly to determine if the incorporation of pHrodo in the porous shell of the particle could modify the toxicological data of the native core-shell particles (without the $\mathrm{pH}$ probe).

The toxicological parameters investigated in this study are described in Fig. 5. In each case, size equivalent particles $(500 \mathrm{~nm}$ or $250 \mathrm{~nm}$ ) were compared for conditions with or without pHrodo probe.

Fig. 5a clearly shows a nonsignificant variation in TNF- $\alpha$ production triggered by the incubation with the particles with either FITC or FITC/pHrodo independent of the size tested. We cannot conclude whether TNF- $\alpha$ production is dependent on sizes of the particles because analyses were performed with an equivalent number of particles in each condition; i.e., with different particle volumes and surfaces.

The different amount of released LDH between FITC and FITC/pHrodo particles (Fig. 5b) was not significant with $250 \mathrm{~nm}$ particles but was significantly enhanced with $500 \mathrm{~nm}$ particles.

Concerning the oxidative stress (Fig. 5c and 5d), results indicated no significant variations for the $500 \mathrm{~nm}$ and $250 \mathrm{~nm}$ particles, irrespective of the presence of pHrodo in the porous shell. After 24 hour exposure, total ROS generation was enhanced comparatively to control cells in all tested conditions, 
probably due to $\mathrm{H}_{2} \mathrm{O}_{2}$, which was likely the source of other organo-peroxides or led to formation of hydroxides.

Regarding these toxicological data, the $250 \mathrm{~nm}$ particles showed no significant differences between the FITC and FITC/pHrodo conditions. In this case, incorporation of pHrodo in the porous shell of the particle seems to have had no significant biological impact. With regard to the $500 \mathrm{~nm}$ particles, only the LDH parameter (membrane alteration) was significantly different between the two types of particles. However, only a slight increase was observed for the double fluorescent particles, and the LDH level was significantly lower than the positive control (i.e., lysed cells). These results may be explained by the ratio that was used and the inherent porosity of the FITC/pHrodo particles. Indeed, in this study the dose of particles was fixed at 1000 particles for one cell irrespective of the size of the particle $(250$ or $500 \mathrm{~nm})$, leading to a different surface area for the two kinds of particles. The $500 \mathrm{~nm}$ particles have a fourfold greater surface area and an eightfold greater volume than the $250 \mathrm{~nm}$ particles. Moreover, pHrodo is integrated in a porous polysiloxane shell. These two parameters, surface volume and porosity, could explain an increase in membrane alterations (LDH parameter). This assumption is further supported by our semi-quantitative data showing no differences in terms of internalization for the two investigated sizes $(89 \pm 2 \%$ for $250 \mathrm{~nm}$ particles vs. $86 \pm 5 \%$ for $500 \mathrm{~nm}$ particles). The double fluorescent particles appeared to be nontoxic for $250 \mathrm{~nm}$ size in our experimental conditions (i.e., 1000/1 cell ratio) and permitted efficient quantification of the uptake. 


\section{Conclusion}

This study was aimed at developing double fluorescent particles for biological investigations, particularly for the study of phagocytosis. We presented a proofof-concept that was first validated in acellular and then in cellular conditions. These particles, whose physicochemical characteristics were well defined and controlled (fluorescence, size, surface coating, state of agglomeration), were nontoxic as determined by various biological parameters, particularly for the 250 nm sized ones. This model of particles could be a powerful tool in biology, one which may be helpful for a better understanding of uptake mechanisms, a key issue of nanotoxicology.

The importance of the physicochemical properties of the particles on biological toxicity is generally described in the literature (Bruch et al. 2004; Clift et al. 2008; Donaldson and Stone 2003; Fubini et al, 2004; Hamilton et al. 2008; Sayes et al 2007; Warheit 2010; Warheit et al. 2007). Further investigations will be conducted with these FITC/pHrodo particles to better understand the impact of their physicochemical properties on the degree of internalization and the cell reactions. This model of particles allows us to modify one by one all the physicochemical parameters: size, functionalized groups (e.g., carboxylate, amine and polyethylene glycol) indirectly indexed to zeta potential modifications reflecting particles surface charges. A key issue will be the synthesis of smaller particles at the nanoscale.

\footnotetext{
Acknowledgements We are grateful to the CMES (Centre de Microscopie Electronique Stéphanois), especially Isabelle Anselme-Bertrand and Charlotte Delorme for the TEM images.
} 


\section{References}

Abedini F, Hosseinkhani H, Ismail M et al (2011) In vitro intracellular trafficking of biodegradable nanoparticles dextran-spermine in cancer cell lines. Int J Nanotechnol 8:712-723. Bos H, de Souza W (2000) Phagocytosis of yeast: a method for concurrent quantification of binding and internalization using differential interference contrast microscopy. J Immunol Methods 28:29-43.

Broz P, Driamov S, Ziegler J et al (2006) Toward intelligent nanosize bioreactors: a pHswitchable, channel-equipped, functional polymer nanocontainer. Nano Lett 6:2349-2353. Bruch J, Rehn S, Rehn B et al (2004) Variation of biological responses to different respirable quartz flours determined by a vector model. Int J Hyg Environ Health 207:203-216.

Buck SM, Xu H, Brasuel M et al (2004) Nanoscale probes encapsulated by biologically localized embedding (PEBBLEs) for ion sensing and imaging in live cells. Talanta 63:41-59.

Catelas I, Petit A, Marchand R et al (1999) Cytotoxicity and macrophage cytokine release induced by ceramic and polyethylene particles in vitro. J Bone Joint Surg Br 81:516-521.

Chithrani DB (2011) Optimization of bio-nano interface using gold nanostructures as a model nanoparticle system. Insciences J, 1(3), 115-135.

Cho EC, Xie J, Wurm PA et al (2009) Understanding the role of surface charges in cellular adsorption versus internalization by selectively removing gold nanoparticles on the cell surface with a I2/KI etchant. Nano Lett 9:1080-1084.

Clark, HA, Kopelman R, Tjalkens R et al (1999) Optical nanosensors for chemical analysis inside single living cells. 2. Sensors for $\mathrm{pH}$ and calcium and the intracellular application of PEBBLE sensors. Anal Chem 71:4837-4843.

Clift MJ, Rothen-Rutishauser B, Brown DM et al (2008) The impact of different nanoparticle surface chemistry and size on uptake and toxicity in a murine macrophage cell line. Toxicol Appl Pharmacol 232:418-427.

De la Harpe J, Nathan CF (1985) A semi-automated micro-assay for $\mathrm{H} 2 \mathrm{O} 2$ release by human blood monocytes and mouse peritoneal macrophages. J Immunol Methods 78:323-336.

Di Palma F, Chamson A, Lafage-Proust MH et al (2004) Physiological strains remodel extracellular matrix and cell-cell adhesion in osteoblastic cells cultured on alumina-coated titanium alloy. Biomaterials 25:2565-2575.

Donaldson K, Brown D, Clouter A et al (2002) The pulmonary toxicology of ultrafine particles. J Aerosol Med 15:213-220.

Donaldson K, Stone V (2003) Current hypotheses on the mechanisms of toxicity of ultrafine particles. Ann Ist Super Sanita 39:405-410.

Fizet J, Rivière C, Bridot JL et al (2009) Multi-luminescent hybrid gadolinium oxide nanoparticles as potential cell labeling. J Nanosci Nanotechnol 9:5717-5725.

Fubini B (1998) Surface chemistry and quartz hazard. Ann Occup Hyg 42:521-530.

Fubini B, Fenoglio I, Ceschino R et al (2004) Relationship between the state of the surface of four commercial quartz flours and their biological activity in vitro and in vivo. Int J Hyg Environ Health 207:89-104.

Fubini B, Ghiazza M, Fenoglio I (2010) Physico-chemical features of engineered nanoparticles relevant to their toxicity. Nanotoxicology 4:347-363.

Griffiths G (2004) On phagosome individuality and membrane signalling networks. Trends Cell Biol 14:343-351.

Hamilton RF, Thakur SA, Holian A (2008) Silica binding and toxicity in alveolar macrophages. Free Radic Biol Med 44:1246-1258.

Harvey BP, Quan TE, Rudenga BJ et al (2008) Editing antigen presentation: antigen transfer between human B lymphocytes and macrophages mediated by class A scavenger receptors. $\mathrm{J}$ Immunol 181:4043-4051.

Hosseinkhani H, Tabata Y (2006) Self assembly of DNA complexes with polycations for the delivery of genetic materials into cells. J Nanosci Nanotechnol 6:1-9.

Hosseinkhani H, Hosseinkhani M, Gabrielson NP et al (2008) DNA nanoparticles encapsulated in 3D tissue-engineered scaffolds enhance osteogenic differentiation of mesenchymal stem cells. J Biomed Mater Res Part A 85A:47-60.

Kobayashi S, Kojidani T, Osakada H et al (2010) Artificial induction of autophagy around polystyrene beads in nonphagocytic cells. Autophagy 6:36-45.

Leclerc L, Boudard D, Pourchez J et al (2010) Quantification of microsized fluorescent particles phagocytosis to a better knowledge of toxicity mechanisms. Inhal Toxicol 22:1091-1100.

Lee YE, Smith R, Kopelman R (2009) Nanoparticle PEBBLE sensors in live cells and in vivo. Annu Rev Anal Chem (Palo Alto Calif) 2:57-76.

Miksa M, Komura H, Wu R et al (2009) A novel method to determine the engulfment of apoptotic cells by macrophages using pHrodo succinimidyl ester. J Immunol Methods 342:71-77. 
Moore NM, Sheppard CL, Barbour TR et al (2008) The effect of endosomal escape peptides on in vitro gene delivery of polyethylene glycol-based vehicles. J Gene Med 10:1134-1149.

Munoz Javier A, Kreft O, Semmling M et al (2008) Uptake of colloidal polyelectrolyte-coated particles and polyelectrolyte multilayer capsules by living cells. Adv Mater 20:4281-4287.

Nan A, Bai X, Son SJ, et al (2008) Cellular uptake and cytotoxicity of silica nanotubes. Nano Lett 8:2150-2154.

Oberdorster G, Oberdorster E, Oberdorster J (2005) Nanotoxicology: an emerging discipline evolving from studies of ultrafine particles. Environ Health Perspect 113:823-839.

Peng J, He X, Wang K et al (2007) Noninvasive monitoring of intracellular $\mathrm{pH}$ change induced by drug stimulation using silica nanoparticle sensors. Anal Bioanal Chem 388:645-654.

Reibetanz U, Chen MH, Mutukumaraswamy S et al (2010) Colloidal DNA carriers for direct localization in cell compartments by $\mathrm{pH}$ sensoring. Biomacromolecules 11:1779-1784.

Reibetanz U, Halozan D, Brumen M et al (2007) Flow cytometry of HEK 293T cells interacting with polyelectrolyte multilayer capsules containing fluorescein-labeled poly(acrylic acid) as a $\mathrm{pH}$ sensor. Biomacromolecules 8:1927-1933.

Romero G, Estrela-Lopis I, Zhou J et al (2010) Surface engineered poly(lactide-co-glycolide) nanoparticles for intracellular delivery: Uptake and cytotoxicity - A confocal raman microscopic study. Biomacromolecules 11:2993-2999.

Sayes CM, Reed KL, Warheit DB (2007) Assessing toxicity of fine and nanoparticles: comparing in vitro measurements to in vivo pulmonary toxicity profiles. Toxicol Sci 97:163-180.

Semmling M, Kreft O, Munoz Javier A et al (2008) A novel flow-cytometry-based assay for cellular uptake studies of polyelectrolyte microcapsules. Small 4:1763-1768.

Sjöback R, Nygren J, Kubista M (1995) Absorption and fluorescence properties of fluorescein. Spectrochimica Acta Part A 51:L7-L21.

Skirtach AG, Munoz Javier A, Kreft O et al (2006) Laser-induced release of encapsulated materials inside living cells. Angew Chem Int Ed Engl 45:4612-4617.

Strunnikova NV, Barb J, Sergeev YV et al (2009) Loss-of-function mutations in Rab escort protein 1 (REP-1) affect intracellular transport in fibroblasts and monocytes of choroideremia patients. PLoS One 4:e8402.

Subramani K, Hosseinkhani H, Khraisat A et al (2009) Targeting nanoparticles as drug delivery systems for cancer treatment. Curr Nanosci 5:135-140.

Van Blaaderen A, Vrij A (1992) Synthesis and characterization of colloidal dispersions of fluorescent, monodisperse silica spheres. Langmuir 8:2921-2931.

Vieira OV, Bucci C, Harrison RE et al (2003) Modulation of Rab5 and Rab7 recruitment to phagosomes by phosphatidylinositol 3-kinase. Mol Cell Biol 23:2501-2514.

Wang W, Gu B, Liang L et al (2003) Fabrication of two- and three-dimensional silica nanocolloidal particle arrays. J Phys Chem B, 107:3400-3404.

Warheit DB (2008) How meaningful are the results of nanotoxicity studies in the absence of adequate material characterization? Toxicol Sci 101:183-185.

Warheit DB (2010) Debunking some misconceptions about nanotoxicology. Nano Lett 10:47774782.

Warheit DB, Laurence BR, Reed KL et al (2004) Comparative pulmonary toxicity assessment of single-wall carbon nanotubes in rats. Toxicol Sci 77:117-125.

Warheit DB, Webb, TR, Colvin VL et al (2007) Pulmonary bioassay studies with nanoscale and fine-quartz particles in rats: toxicity is not dependent upon particle size but on surface characteristics. Toxicol Sci 95:270-280. 


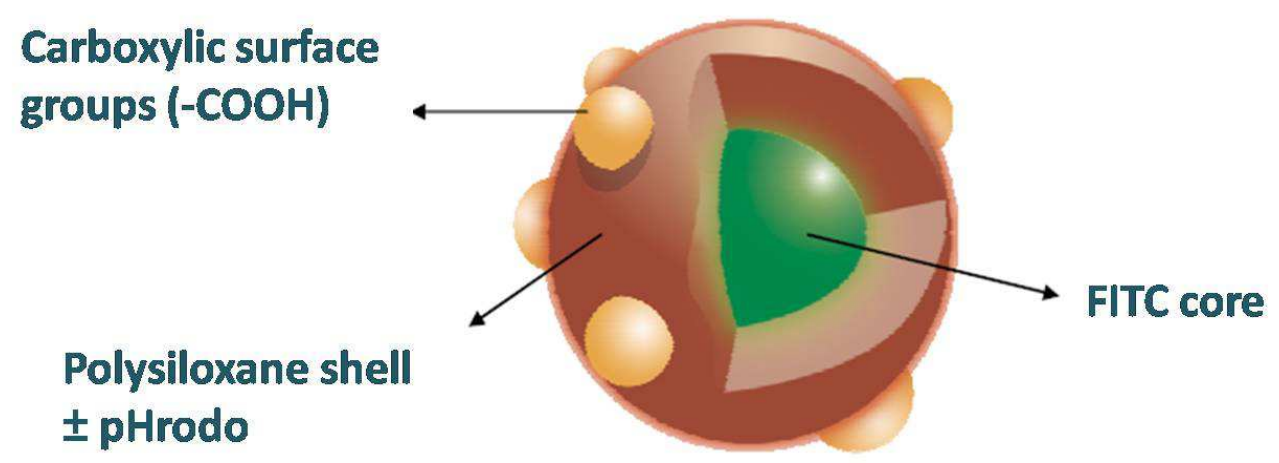

Fig. 1 Schematic representation of the particles developed. Two types were designed: FITC particles (FITC core, polysiloxane shell and $\mathrm{COOH}$ surface groups) and FITC/pHrodo particles (FITC core, porous polysiloxane shell functionalized with $\mathrm{pHrodo}^{\mathrm{TM}}$ probe and $\mathrm{COOH}$ surface groups). FITC particles have a single green fluorescence. In acidic conditions FITC/pHrodo particles have a double fluorescence (green for FITC core and red for pHrodo probe). Excitation and emission wavelengths were $488 \mathrm{~nm}$ and 495-550 nm for FITC and 532 and 560-650 nm for pHrodo, respectively 

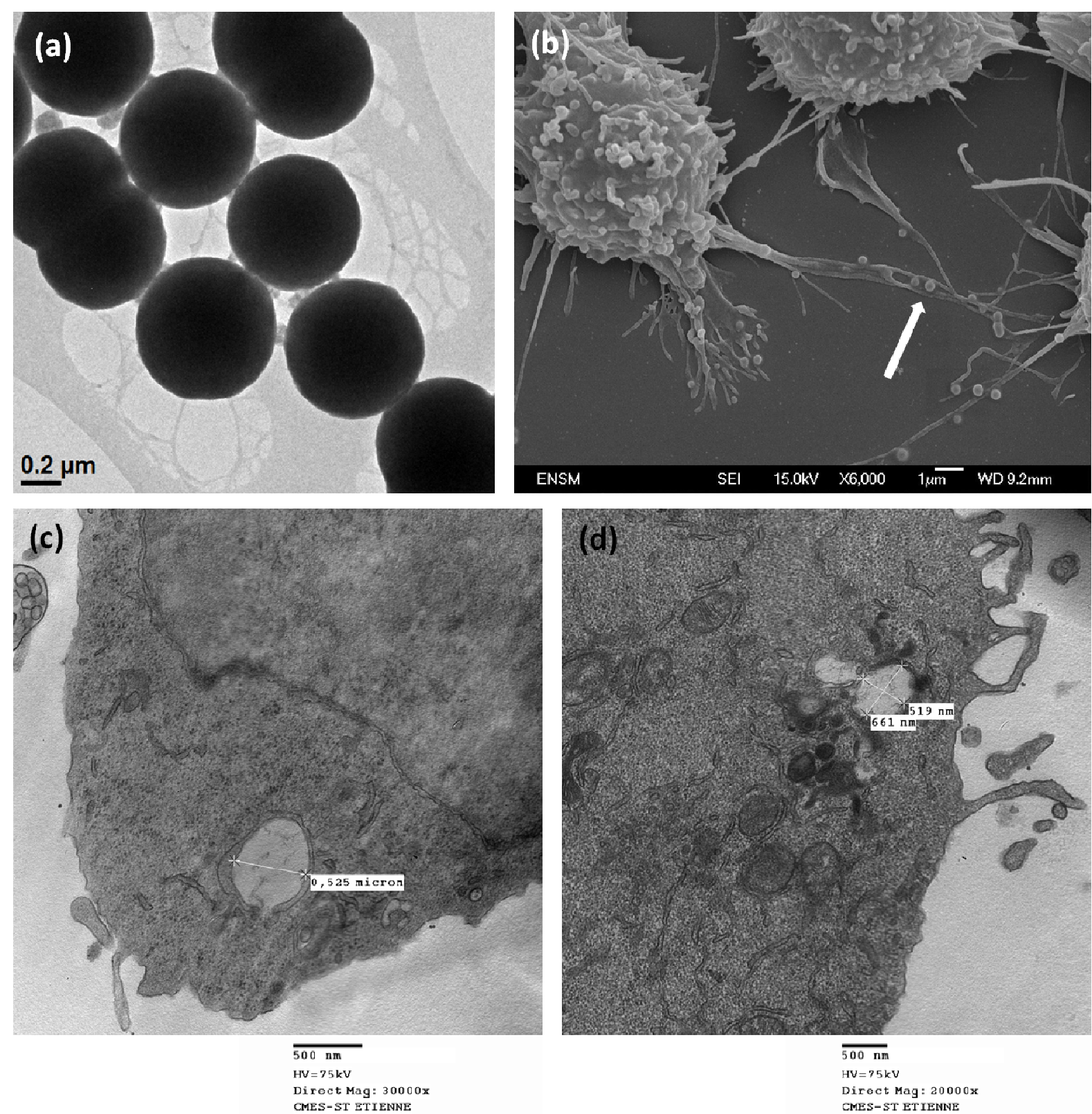

Fig. 2 Electron microscopy observations of $500 \mathrm{~nm}$ FITC particles. a TEM of particles alone, $\mathbf{b}$ SEM after a 24 hour contact with macrophages RAW 264.7 and $\mathbf{c}, \mathbf{d}$ TEM after 24 hour contact with macrophages RAW $264.7(\mathrm{HV}=75 \mathrm{kV})$ 


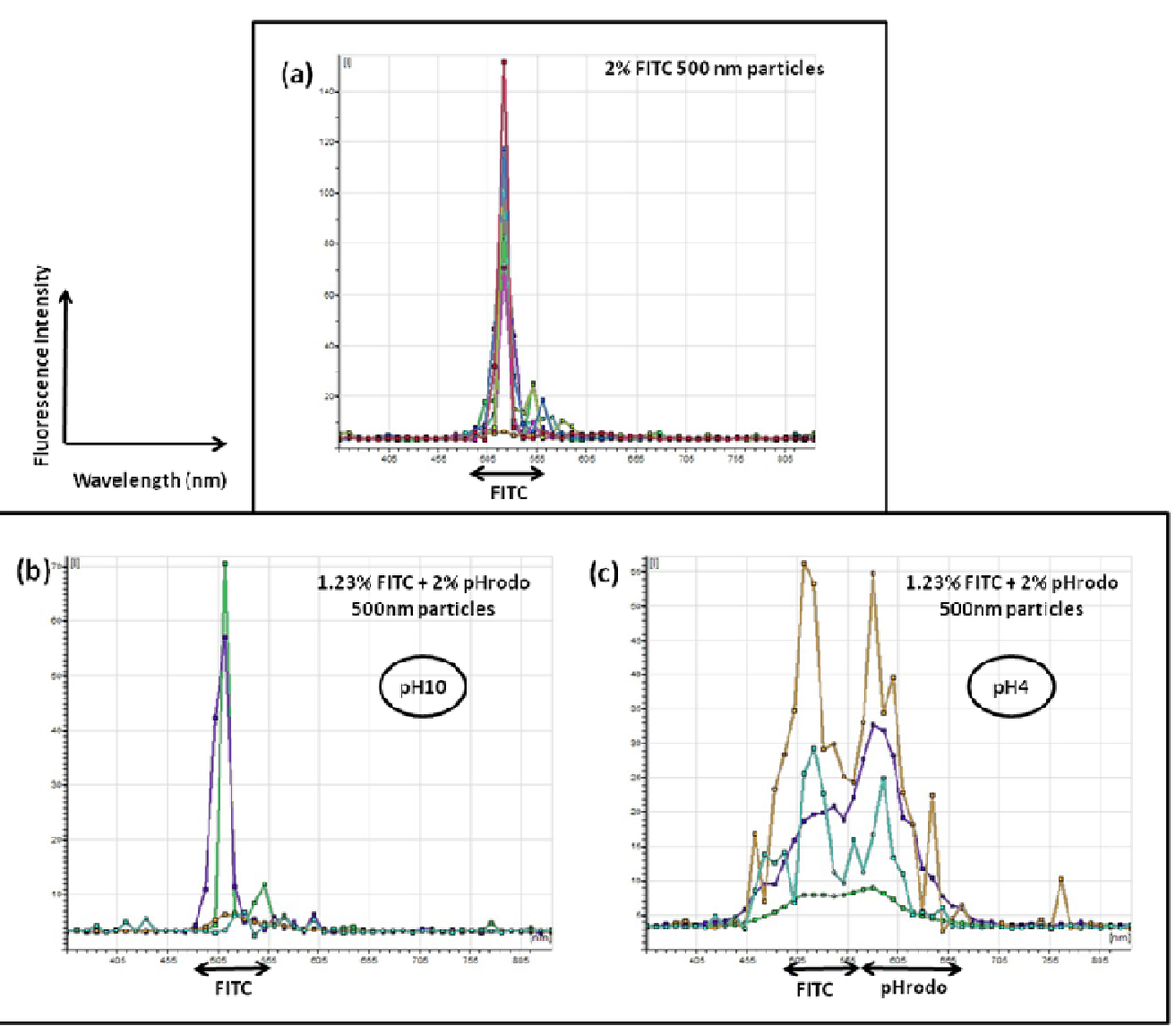

Fig. 3 Fluorescence spectra acquired by confocal microscopy in acellular conditions for $500 \mathrm{~nm}$ $2 \%$ FITC particles (a) and for $1.23 \%$ FITC/2\% pHrodo particles at pH 10 (b) and $\mathrm{pH} 4$ (c). Intensity of pHrodo fluorescence increases as $\mathrm{pH}$ decreases. A mild FITC fluorescence intensity decrease is observed between the two types of particles. Each curve corresponds to different regions of interest positioned on image stacks acquired during the confocal spectra scan 


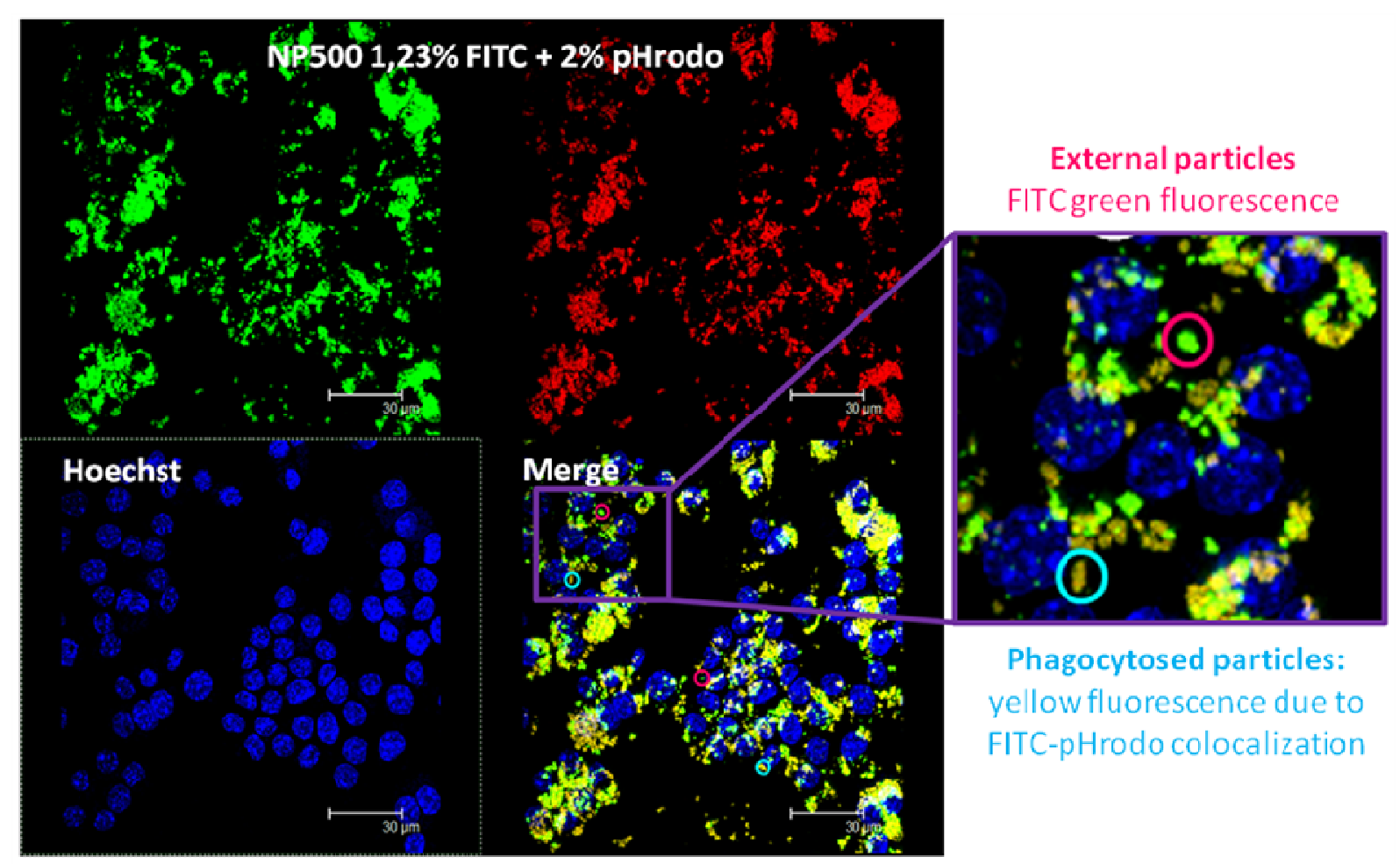

Fig. 4 Example of confocal microscopy acquisition of $500 \mathrm{~nm}$ FITC/pHrodo particles after 24 hour contact with RAW 264.7 macrophages. Cell nuclei were stained with Hoechst (blue). The enlargement allows the distinction between external particles (green) and phagocytosed particles (yellow due to the merge of green and red fluorescence). Excitation and emission wavelengths, respectively, for FITC (488 nm, 495-550 nm), pHrodo (532 nm, 560-650 nm) and Hoechst (350 $\mathrm{nm}, 408-443 \mathrm{~nm}$ ) 
(a)

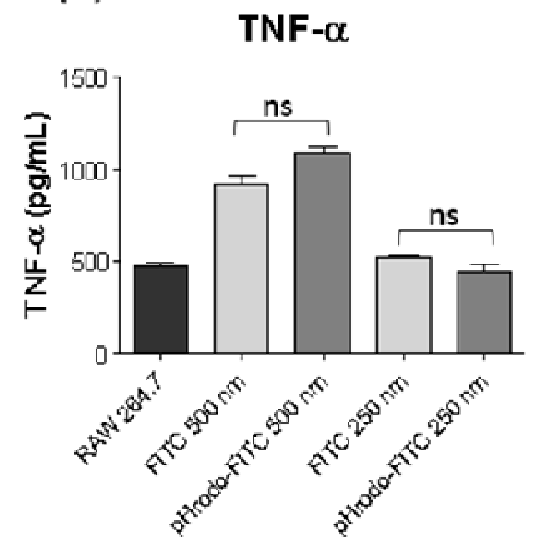

(c)

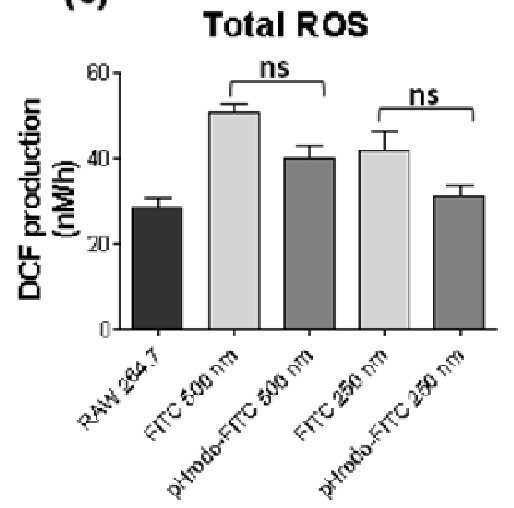

(b)

\section{LDH}

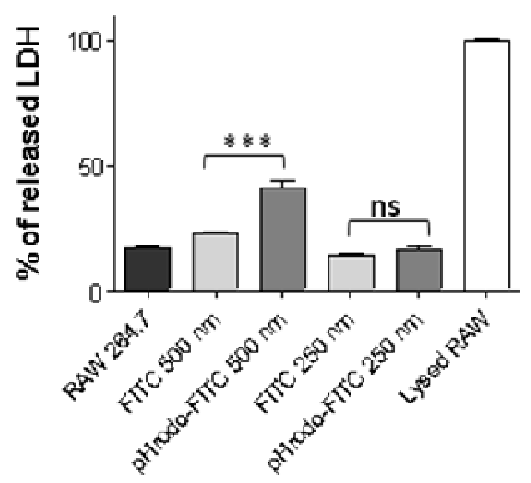

(d)

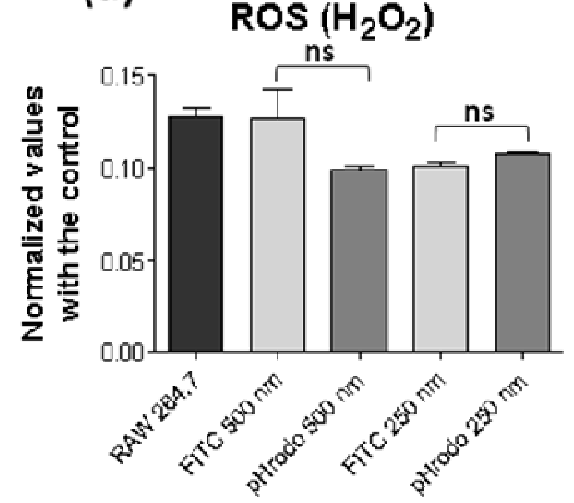

Fig. 5 Toxicological analysis of single or double fluorescent particles (FITC or FITC/pHrodo particles) for 500 or $250 \mathrm{~nm}$ respectively incubated with RAW 264.7 cells for 24 hours (1000 particles/cell). a TNF- $\alpha$ production, $\mathbf{b}$ percentage of released LDH, $\mathbf{c}$ total ROS generated and $\mathbf{d}$ $\mathrm{H}_{2} \mathrm{O}_{2}$ produced $(n=3, * p<0.05)$

Table 1 Particle size evaluated by TEM and size distribution - zeta potential measured by DLS for particles in water or in complete culture media

\begin{tabular}{|c|c|c|c|c|c|}
\cline { 2 - 6 } \multicolumn{1}{c|}{} & \multicolumn{3}{c|}{ Size characterization (nm) } & \multicolumn{2}{c|}{ Zeta Potential } \\
\hline Particles types and expected sizes & TEM & DLS - Water & DLS - Culture media & Water & Culture media \\
\hline FITC particles - 500 nm & $411 \pm 23$ & $434 \pm 11$ & $492 \pm 6$ & $-65.6 \pm 0.3$ & $-22.6 \pm 2.2$ \\
\hline FITC/pHrodo particles - 500 nm & $492 \pm 19$ & $512 \pm 6$ & $514 \pm 8$ & $-57.6 \pm 0.5$ & $-16.2 \pm 0.7$ \\
\hline FITC particles - 250 nm & $240 \pm 17$ & $269 \pm 10$ & $279 \pm 4$ & $-54.5 \pm 0.9$ & $-24.5 \pm 1.5$ \\
\hline pHrodo/FITC particles - 250 nm & $224 \pm 15$ & $307 \pm 9$ & $232 \pm 2$ & $-53.4 \pm 1.7$ & $-19 \pm 0.7$ \\
\hline
\end{tabular}



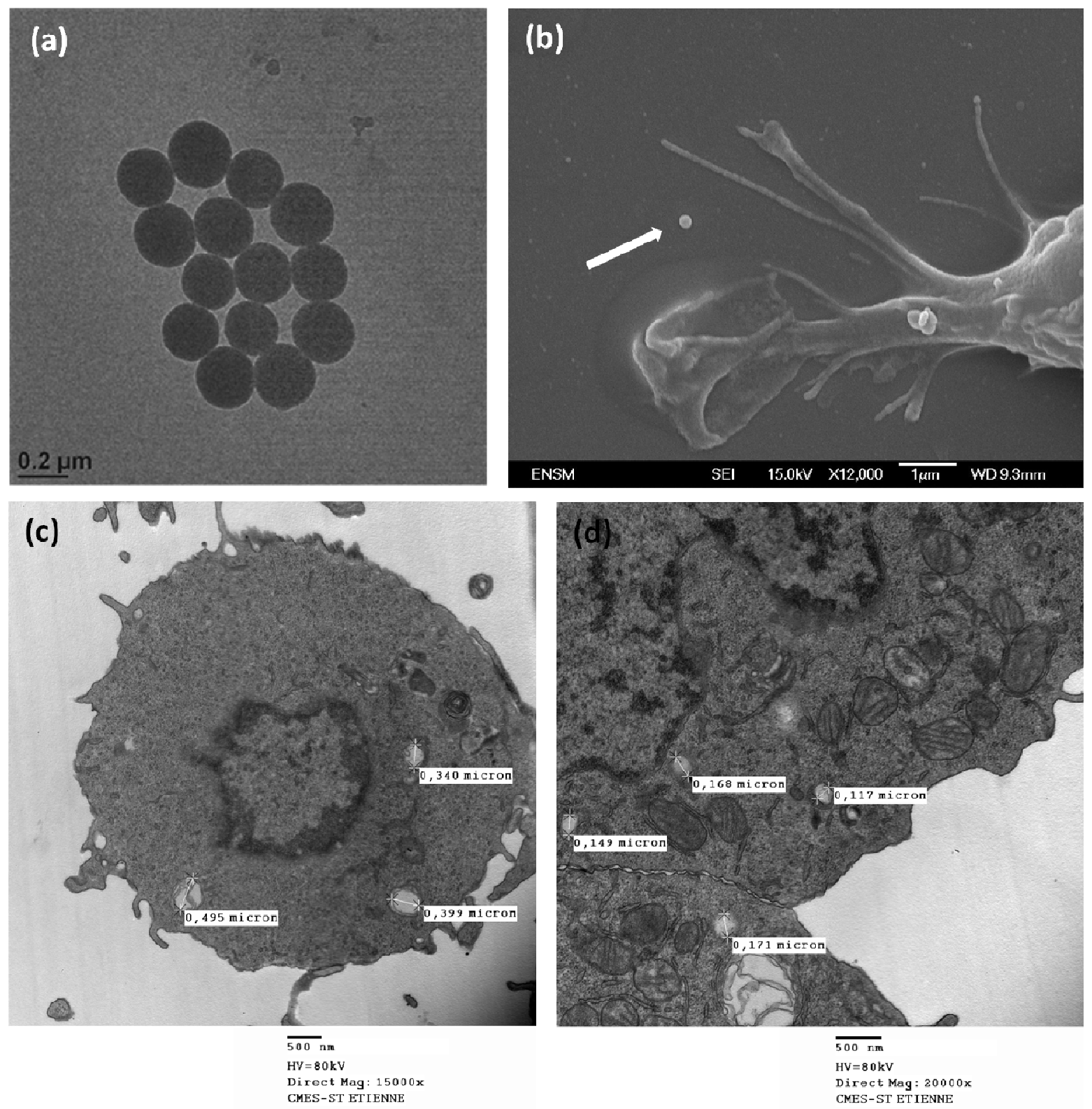

Supplementary Fig. 1 Electron microscopy observations of $250 \mathrm{~nm}$ FITC particles. a TEM of particles alone, b SEM after a 24 hour contact with macrophages RAW 264.7 and $\mathbf{c}, \mathbf{d}$ TEM after 24 hour contact with macrophages RAW $264.7(\mathrm{HV}=80 \mathrm{kV})$ 


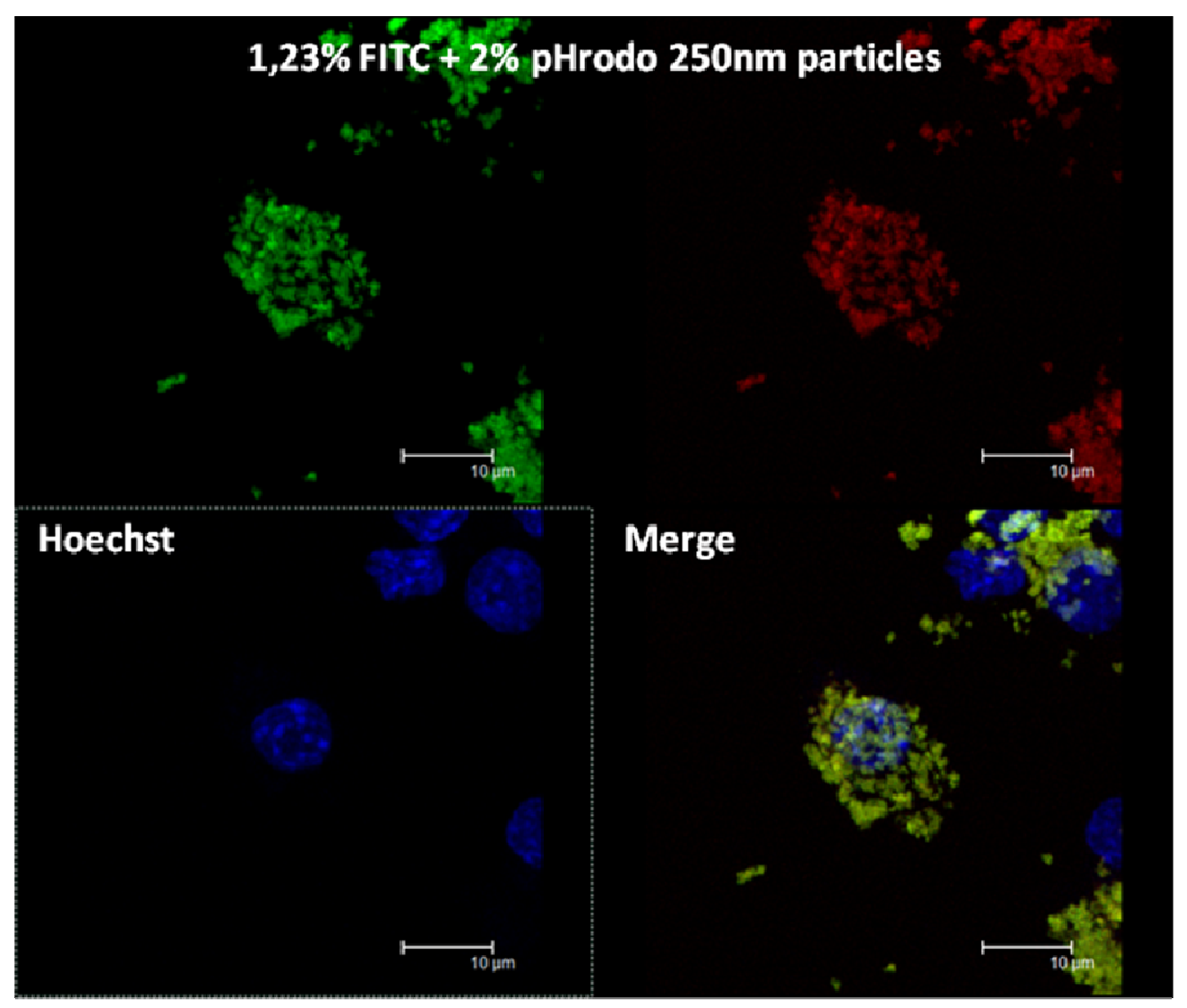

Supplementary Fig. 2 Example of confocal microscopy acquisition of $250 \mathrm{~nm}$ FITC/pHrodo particles after 24 hour contact with RAW 264.7 macrophages. Cell nuclei were stained with Hoechst (blue). Distinction is observed between external particles (green) and phagocytosed particles (yellow due to the merge of green and red fluorescences). Excitation and emission wavelengths, respectively, FITC $(488 \mathrm{~nm}, 495-550 \mathrm{~nm})$, pHrodo $(532 \mathrm{~nm}, 560-650 \mathrm{~nm})$ and Hoechst (350 nm, 408-443 nm) 
Supplementary Table. Fluorescence optimization of dye amount. The FITC or pHrodo photobleachning was assessed under the confocal beam. Bleaching: low $(+)$, high $(++)$, strong $(+++)$ or no bleaching low (-), high(--) or strong(---) were estimated for the two sizes of particles. The best yield was obtained for $1.23 \%$ FITC and $2 \%$ pHrodo

\begin{tabular}{|c|c|c|c|c|c|}
\hline $\begin{array}{c}\text { Particles } \\
\text { suspensions }\end{array}$ & $\begin{array}{c}\text { Expected } \\
\text { sizes } \\
(\mathbf{n m})\end{array}$ & $\begin{array}{c}\% \\
\text { FITC }\end{array}$ & $\begin{array}{c}\% \\
\text { pHrodo }\end{array}$ & $\begin{array}{c}\text { FITC } \\
\text { bleaching }\end{array}$ & $\begin{array}{c}\text { pHrodo } \\
\text { bleaching }\end{array}$ \\
\hline FITC & 500 & $1.23 \%$ & $0 \%$ & -- & $*$ \\
\hline FITC & 500 & $3 \%$ & $0 \%$ & --- & $*$ \\
\hline FITC & 500 & $5 \%$ & $0 \%$ & --- & $*$ \\
\hline FITC & 250 & $2 \%$ & $0 \%$ & --- & $*$ \\
\hline FITC & 250 & $3 \%$ & $0 \%$ & --- & $*$ \\
\hline FITC/pHrodo & 500 & $2 \%$ & $<0.5 \%$ & --- & +++ \\
\hline FITC/pHrodo & 500 & $1.23 \%$ & $0.5 \%$ & -- & + \\
\hline FITC/pHrodo & 500 & $1.23 \%$ & $2 \%$ & -- & - \\
\hline FITC/pHrodo & 250 & $2 \%$ & $0.1 \%$ & --- & +++ \\
\hline FITC/pHrodo & 250 & $2 \%$ & $0.2 \%$ & --- & +++ \\
\hline FITC/pHrodo & 250 & $2 \%$ & $0.3 \%$ & --- & ++ \\
\hline FITC/pHrodo & 250 & $2 \%$ & $0.5 \%$ & --- & + \\
\hline FITC/pHrodo & 250 & $1.23 \%$ & $0.5 \%$ & -- & + \\
\hline FITC/pHrodo & 250 & $1.23 \%$ & $2 \%$ & -- & - \\
\hline
\end{tabular}

